\title{
Techniques of robot-assisted kidney transplantation
}

\author{
Seoungjun Lim, Youngmin Ko, Donghyun Kim, Joohee Jung, Hyunwook Kwon, Younghoon Kim, Duckjong Han, Sung Shin
}

Division of Transplantation, Department of Surgery, Asan Medical Center, University of Ulsan College of Medicine, Seoul, Korea

Background: This article shows the details of robot-assisted kidney transplantation (RAKT) from a living donor. RAKT was performed with Da Vinci Si.

Methods: The patient was placed supine with the legs parted and in Trendelenburg position. The Da Vinci robot was docked between the legs. Kidney allograft was given from a living donor. Before vascular anastomosis, a kidney allograft was prepared on the back table including insertion of a double-J stent in the ureter. The kidney allograft was wrapped in an ice-packed gauze to lower the temperature during the anastomosis time. A 12-mm port for robotic camera, three 8-mm ports for robotic arms, and a 12-mm port for an assistant were placed. After creating peritoneal pouch for the kidney allograft, dissection of iliac vessels and bladder was performed. Through a $6-\mathrm{cm}$ Pfannenstiel incision, the kidney was inserted into the peritoneal pouch lateral to right iliac vessels. After the external iliac vein was clamped with Bulldogs clamps, a venotomy was given and the graft renal vein was anastomosed to the external iliac vein in an end-to-side continuous manner with a 6/0 GORE-TEX CV-6 (W.L. Gore and Associates Inc., Flagstaff, AZ, USA). After the graft renal vein was clamped, the iliac vein was declamped. Similarly, clamping of the external iliac artery, arteriotomy, arterial anastomosis with a 6/0 GORE-TEX CV-6, clamping of the graft renal artery, and declamping of the external iliac artery were performed. Reperfusion was done and ureteroneocystostomy was performed according to the Lich-Gregoir technique. Jackson-Pratt drain was placed through one of working ports and the peritoneum was closed at a few locations with Hem-o-lok.

Results: Three patients were operated with RAKT. The mean surgery duration was 441 minutes. The mean postoperative hospital days were 7.3 days. The mean of lowest post-operation creatine was $1.38 \mathrm{mg} / \mathrm{dL}$.

Conclusions: RAKT could be an alternative method in kidney transplantation.

Corresponding author: Seoungjun Lim

E-mail: dkhe1986@naver.com

(c) The Korean Society for Transplantation

This is an Open Access article distributed under the terms of the Creative Commons Attribution Non-Commercial License (http://creativecommons.org/licenses/by-nc/4.0/) which permits unrestricted non-commercial use, distribution, and reproduction in any medium, provided the original work is properly cited. 